\section{The Transmission Confocal Laser Scanning Microscope}

David Carter

University of Western Ontario

A confocal laser scanning microscope which can collect images in both transmission and reflection modes has been installed and is being tested in the Imaging Laboratories of the John P. Roberts Research Institute, London, Ontario. Designed by $\mathrm{Dr}$. Ted Dixon at the University of Waterloo, it is being developed by a multi-disciplinary research group which includes the Ontario Lasers and Lightwaves Research Centre and the Department of Physiology, University of Toronto; the Radiology. Physics, and Pathology Departments, MacMaster University; and the Zoology Department, University of Western Ontario.

Commercial confocal microscopes operate by reflectance or epifluorescence. A pair of scanning mirrors direct a diffuse laser beam in a raster pattern through an objective lens, which focuses it on the specimen. Reflected light passes back along the same light path, being "de-scanned" by the moving mirrors and then diverted by a beam splitter into a detector. On its return to the detector, light from the focal plane is focussed through a pinhole, which blocks light from out-of-focus regions of the spectrum. The microscope stage is moved up and down by a stepping motor to collect images at different depths.

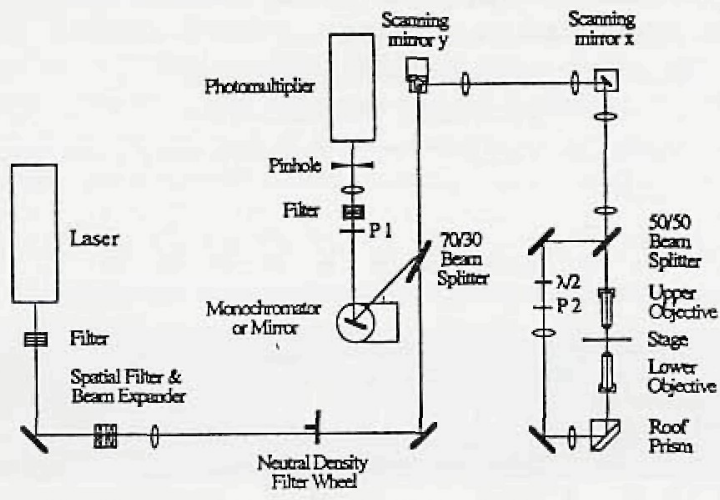

The new instrument has a transmission arm which aligns an additional objective lens under the specimen (refer above). This has a roof prism which inverts the raster pattern so that both objectives scan points in the specimen in the same order. It also has a pair of lenses to keep all beams diverging at the same rate, when merged at the specimen and the beam splitter. The two objectives are aligned so that each acts as a condenser to the other for making transmission confocal images, and so that reflection and transmission images are all in register.

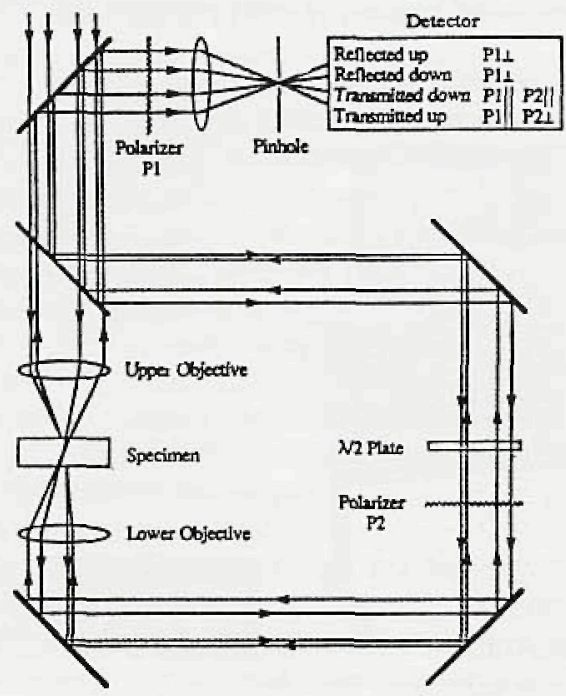

Four possible light paths can be isolated and viewed separately or in combination (refer previous). Light producing transmission images from either direction pass though the $\lambda / 2$ plate once, so their plane of polarization is rotated through $90^{\circ}$ relative to the ordinal laser beam. Light producing reflection images retain the polarity of the original laser beam because the upper reflection image does not pass the $\lambda / 2$ plate, while the lower reflection image passes through the $\lambda / 2$ plate twice. Switching the polarized in front of the detector from perpendicular to parallel, selects transmission and reflection modes respectively.

A reflection image from just one side is selected by covering one of the objective lenses. A single direction of transmission is selected by adding a second polarizing filter below the $\lambda / 2$ plate. Here, the transmitted image formed in the downward direction has a parallel polarization, while light making the tansmitted image in the upward direction has been rotated through $90^{\circ}$. Omission of this polarizing filter results in a combined image from light transmitted in both directions, while removal of the detector filter produces an image combining all four light paths. By setting the polarizing filters to intermediate values, it may be possible to combine the signals in any desired ratio.

The microscope is built on a vertical $3^{\prime} \times 3^{\prime}$ breadboard, mounted on a $4^{\prime} \times 6^{\prime}$ vibration isolated optical table. The open plan design makes it easy to change its configuration and to integrate it with other equipment. In its present form, our instrument is powered by an air cooled $50 \mathrm{~mW}$ Argon lon laser. It has a pair of Mititoyo x100 NA 0.7 dry objective lenses, which can sample a $12 \mathrm{~mm}$ depth of field at $0.1 \mu \mathrm{m}$ increments, and give a field of view $200 \mu$ wide. The sampled area can be reduced to $100 \mu$ or $50 \mu$, to increase magnification by two or four times. A diffraction grating monochromator on a rotating stage is used to select any wavelength for detection, with a precision of $1 \mathrm{~nm}$. To increase signal intensity, this can be rep;aced with a mirror.

In a conventional confocal microscope, deeper layers of the specimen are partially obscured by opaque or refractive material in overlying layers. This results in a reduction in resolution with depth of penetration, the casting of shadows by opaque regions, and production of image ghosts by refractive or reflective layers with the specimen. In transmission mode, all layers of the specimen are illuminated equally, which should help to reduce these artifacts. However, transmission confocal images look very different from those from epifluorescent machines because contrast is produced in a different way, so great care is needed in their interpretation.

A transmission confocal instrument is not restricted to the exclusive use of epifluorescence. Anything which absorbs or reflects at least one of the laser frequencies could be used to generate tansmission confocal images. In transmission mode, we can use a whole spectrum of non-fluorescent stains which were previously limited to the study of thin sections. This opens the possibility of spectroscopic confocal observation of archival histological specimens. We may also be able to use several dyes simultaneously to measure different parameters in the same cells at the same time.

Physiological conditions within living cells can be measured using a range of fluorescent reagents and a flow cyclometer. Examples include membrane potentials (OxonolVI), $\mathrm{pH}$ levels (BCECF), and ion concentrations (FURA2, Calcium; Mag-FURA2, Magnesium; SBFI, Sodium; PBFI, Potassium; SPQ, Chloride, etc.). These reagents absorb or fluoresce at two different frequencies, the ratio between them corresponding to the parameter being measured regardless of dye concentration. To date, use of these dyes by microscopists has been limited by the difficulty of incorporating multiple illumination and detection systems, and the necessity to digitize images and calculate image ratios. This will be less of a problem with the new transmission microscope. With its tunable detector and capability for image analysis, it would be an ideal tool for extending the use of these reagents for studying conditions within solid tissues and single cells

The instrument is still in an early stage of development and has yet to prove its potential in the study of biological specimens. However, it has already attracted interest from fields as diverse as silicon chip manufacture, biophysics and the pulp and paper industry. Assuming the present phase of development is successful, we hope to develop a third generation of prototypes to prepare for its development as a commercial product. 